\title{
Performance of Fetal Renal Artery Doppler Compared with Umbilical Artery Doppler in Mild and Moderate Fetal Growth Restriction: An Observational Study in a Tertiary Care Hospital
}

\author{
Anjali Mundkur ${ }^{1}$, Sanghamitra Paladugu², Srikar Yedlapalli ${ }^{3}$, Akhila Vasudeva $^{4}$, Roopa P Shivananda $^{5}$, Nivedita Hegde ${ }^{6}$
}

\begin{abstract}
Background: Fetal growth restriction (FGR) and oligoamnios are one of the major reasons of preterm delivery and low birth weight contributing to almost two-third of neonatal mortality. Fetal kidney, in addition to placenta, acts in controlling and regulating physiology of the fetus. Doppler ultrasound has become a part of routine antenatal care in monitoring such high-risk pregnancies. However, renal artery was the least studied. Aim and objective: To compare the relationship of perinatal outcomes with renal artery Doppler and umbilical artery Doppler indices.

Materials and methods: A prospective observational study carried out among women attending an antenatal clinic and who have undergone delivery in Department of Obstetrics and Gynecology, in a tertiary care hospital between August 2016 and May 2018. Regular ultrasonography was done at 28-31 weeks; they were followed up 4 weeks later and Doppler indices were noted.

Results: In the 394 patients studied, 13.1\% were having FGR, 12.2\% were oligoamnios, and $12.2 \%$ had preterm delivery. The $p$ values of the pulsatility index and the resistivity index of the renal artery in FGR, oligoamnios, preterm delivery, and low birth weight were more significant than umbilical artery Doppler indices.

Conclusion: Renal artery Doppler indices were significantly more reliable in predicting adverse perinatal outcomes in cases of mild uteroplacental insufficiency. Additionally, renal artery Doppler indices were able to pick up these changes earlier than umbilical artery Doppler, hence a potential early indicator, and the pulsatility index being more specific and sensitive.

Limitations: Sample size of the study is limited with only mild cases of FGR. Larger studies are required. In addition, patients at a higher risk of FGR in comparison with low-risk cases should be studied with more sample size as this study did not show any significant association with comorbidities.

Keywords: Doppler, Fetal growth restriction, Fetal renal artery Doppler indices, Intrauterine growth restriction, Oligoamnios, Pulsatility index, Resistivity index, Umbilical artery Doppler indices.

International Journal of Infertility and Fetal Medicine (2020): 10.5005/jp-journals-10016-1194
\end{abstract}

\section{INTRODUCTION}

Fetal growth restriction (FGR) still remains one of the most difficult conundrums in fetal medicine. There are a large number of research articles related to this domain, and yet controversies in diagnosis and management remain. Umbilical artery Doppler and middle cerebral artery Doppler studies have always been the first tests to evaluate blood flow in a growth-restricted fetus. There have been reports in clinical practice, when these Doppler indices individually gave a false impression of fetal compromise leading to untimely and unnecessary interventions.' Umbilical artery Doppler indices also had limited role in predicting perinatal outcome, in fetuses with subtle reduction in growth and liquor. ${ }^{2}$

Fetal kidney, like placenta, has a major physiological role. However, there have been only a few studies on renal artery Doppler and fewer still comparing the umbilical artery and renal artery Doppler. In the present study, perinatal outcomes were correlated with renal artery and umbilical artery Doppler indices and their sensitivities and specificities analyzed.

\section{Materials and Methods}

This was a prospective observational study carried out among women attending antenatal clinic, with informed consent and

\begin{abstract}
${ }^{1-6}$ Department of Obstetrics and Gynecology, Kasturba Medical College and Hospital, Manipal Academy of Higher Education, Manipal, Karnataka, India

Corresponding Author: Sanghamitra Paladugu, Department of Obstetrics and Gynecology, Kasturba Medical College and Hospital, Manipal Academy of Higher Education, Manipal, Karnataka, India, Phone: +91 8296540094, e-mail: mitra.paladugu@gmail.com

How to cite this article: Mundkur A, Paladugu S, Yedlapalli $S$, et al. Performance of Fetal Renal Artery Doppler Compared with Umbilical Artery Doppler in Mild and Moderate Fetal Growth Restriction: An Observational Study in a Tertiary Care Hospital. Int J Infertil Fetal Med 2020;11(1):1-4.
\end{abstract}

Source of support: Nil

Conflict of interest: None

who have undergone delivery in Department of Obstetrics and Gynecology of a tertiary care hospital, between August 2016 and May 2018. This study was intended to establish the correlation between fetal renal artery Doppler, FGR, and to compare it with performance of umbilical artery Doppler. Furthermore, course of events (gestational hypertension, preeclampsia, oligoamnios, late preterm birth) and perinatal outcomes of these pregnancies 
were also correlated with renal artery and umbilical artery Doppler indices. The sample was recruited as per inclusion and exclusion criteria.

\section{Exclusion Criteria}

- Women with multifetal pregnancy

- Women with fetal anomalies/fetal renal anomalies

- Women who had preterm deliveries before 34 weeks

- Women with early-onset FGR at the time of first scan (28-31 weeks)

\section{Sample Size Calculation}

Based on estimated proportions, considering dropouts and lost to follow-up with a $5 \%$ error, the estimated sample size was 384 . A total of 394 patients were recruited who satisfied the inclusion criteria. The research study plan was verified and approved by the institutional ethical committee (IEC NO: 468/2016) prior to data collection.

Ultrasonography was performed for the women at 28-31 weeks and 32-34 weeks as part of routine antenatal care. In the ultrasound, fetal growth parameters, amniotic fluid index, and Doppler were recorded. For purpose of ultrasonography, Voluson P8 with a curvilinear 2.5-MHz probe was used. Both umbilical artery and renal artery Doppler were obtained at the same time. Umbilical artery Doppler was obtained by placing the Doppler sample on the umbilical artery as close to the cord insertion as possible when there is no fetal breathing movement. The renal artery is identified by its origin from the fetal descending aorta. When the fetus remained sufficiently quiet, values are obtained. Renal arteries originate from the aorta at an angle of $70^{\circ}$. Hence, kidney (whichever side is easily obtained in view) is first obtained in a longitudinal axis and renal arteries are traced and the probe is angulated to reduce aortic interference reducing the angle of Doppler sample gate, making it as close to $0^{\circ}$ (was always less than $30^{\circ}$ ) as possible. ${ }^{3}$ Minimum of three values are obtained to reduce bias and the average value is considered. All the scans in this study were performed by a single observer reducing bias. ${ }^{4}$

\section{Statistical Analysis}

Data were analyzed using the software Medcalc version18. Age and weights at birth were analyzed using descriptive statistics (mean and standard deviations). Receiver operator curves were plotted for Doppler indices. They were correlated using the Chi-square test and means were compared using the independent samples $t$-test. Sensitivity and specificity of each Doppler value were estimated and the $p$ value less than 0.05 was considered to be statistically significant.

\section{Results}

Out of 394, 160 were primigravidae, 245 delivered by cesarean section (CS), and 149 delivered vaginally.

The growth parameters, amniotic fluid index, umbilical artery Doppler, and renal artery Doppler at two different gestations, 28-31 weeks and 32-34 weeks, were evaluated. Some patients were excluded from the study either due to preeclampsia, preterm prelabor rupture of the membrane, preterm labor, and intrauterine death.

In the present study, 48 pregnancies were found to be delivered before 37 weeks, 52 had FGR, 48 had oligohydramnios, 73 had low birth weight $(<2.5 \mathrm{~kg}), 27$ babies were admitted to NICU.
At 28-31 weeks, mean pulsatility index (PI) calculated was $3.24 \pm 0.65$, and mean resistivity index (RI) calculated was $0.96 \pm$ 0.15 . At 32-34 weeks scan, mean PI calculated was $2.41 \pm 0.49$, and mean RI was $0.86 \pm 0.09$.

The cutoffs established for PI and RI at 28-31 weeks were 2.8 and 0.91 . The PI at 32-34 weeks could predict FGR. But the RI at 32-34 weeks could not predict FGR and the area under the receiver operating curve (ROC) curve was just above 0.5 , hence cut-off with good sensitivity and specificity could not be established. Mean umbilical artery PI in both the groups was normal even in FGR cases.

The optimal cutoffs established for PI were 3.07 and 2.2 at 28-31 weeks and 32-34 weeks, respectively, analyzed using the ROC. Pulsatility indices of renal artery Doppler evaluated at both gestational ages (as described) could predict oligohydramnios, with higher values indicating high risk.

Renal artery Doppler at 28-31 weeks was able to predict spontaneous preterm birth with good sensitivity and specificity, above the cutoff of $2.8 \mathrm{PI}$ and $0.97 \mathrm{RI}$. The mean PI and RI of renal artery Doppler at 32-34 weeks were $2.2 \pm 0.28$ and $0.85 \pm$ 0.10 . Among the preterm deliveries analyzed, the mean PI was statistically significant for preterm deliveries with $p$ value of 0.04 . The sensitivity and specificity were 56.62 and $71.6 \%$.

The renal artery Doppler PI and RI at 28-31 weeks of normal fetuses when compared with babies who had NICU admissions showed they were statistically significant in predicting the outcome with good sensitivity and specificity of 88 and $73 \%$ for PI. The RI was not found to be statistically significant.

Overall, it was observed that the mean umbilical artery PI and $\mathrm{RI}$ were within normal ranges in all cases, irrespective of normal birth weight, FGR, oligamnios, preterm birth, or low birth weight. There was no statistical correlation with mean PI of the umbilical artery and these cases.

Among 394 patients, 59 patients were found to be under high risk such as previous IUD, abruption, preeclampsia, gestational diabetes, and recurrent pregnancy loss. No significant association was found with renal artery and umbilical artery Doppler indices.

\section{Discussion}

Doppler ultrasonography helped in analyzing fetal hemodynamics. Renal artery Doppler indices and umbilical artery Doppler indices were correlated with perinatal outcomes in fetuses with FGR. Very few studies have correlated performance of renal artery Doppler indices with umbilical artery Doppler indices. The present study shows that renal artery Doppler indices may help predict perinatal outcomes in mild FGR, oligoamnios, NICU admissions, and spontaneous preterm deliveries.

The mean PI and RI (Table 1) values both at 28 weeks and 34 weeks as established in the results were between the 50th and 95th centile of the normal reference ranges established in previous studies by Arduini et al., Andriani et al., and in a study on low-risk Thailand population by Ponglopisit et al. ${ }^{5-7}$

Table 1: Mean renal artery Doppler indices in study population

\begin{tabular}{lll}
\hline Period of gestation & Renal artery Doppler & Mean \pm 2SD \\
\hline 28-31 weeks & $\mathrm{PI}$ & $3.24 \pm 0.65$ \\
& $\mathrm{RI}$ & $0.96 \pm 0.15$ \\
$32-34$ weeks & $\mathrm{PI}$ & $2.41 \pm 0.49$ \\
& $\mathrm{RI}$ & $0.86 \pm 0.09$ \\
\hline
\end{tabular}

$\mathrm{Pl}$, pulsatility index; $\mathrm{Rl}$, resistivity index 
Table 2: Analysis of renal artery Doppler (RAD) and umbilical artery Doppler (UAD) in fetal growth restriction (FGR)

\begin{tabular}{lrlll}
\hline FGR $(n=52)$ & Mean \pm 2 SD & $p$ value & $\begin{array}{l}\text { Sensitivity } \\
(\%)\end{array}$ & $\begin{array}{l}\text { Specificity } \\
(\%)\end{array}$ \\
\hline RAD 28w PI & $2.8 \pm 0.38$ & 0.0001 & 63.46 & 75.15 \\
RI & $0.91 \pm 0.21$ & 0.0042 & 42.31 & 78.95 \\
RAD 34w PI & $2.2 \pm 0.25$ & 0.014 & 53.85 & 78.95 \\
RI & $0.86 \pm 0.11$ & 0.38 & 69.23 & 42.4 \\
UAD 28w PI & $0.87 \pm 0.12$ & 0.35 & 40.38 & 71.93 \\
RI & $0.72 \pm 0.08$ & 0.59 & 55.77 & 53.22 \\
UAD 34w PI & $0.78 \pm 0.12$ & 0.17 & 46.15 & 66.08 \\
RI & $0.65 \pm 0.15$ & 0.057 & 65.38 & 50.29 \\
\hline
\end{tabular}

$\mathrm{Rl}$, resistivity index

The findings of this study are consistent with previous studies that have shown that there is a reduction in $\mathrm{PI}$ and $\mathrm{RI}$ of all fetal vessels and umbilical artery with gestational age. This was explained by the reduced peripheral vascular resistance in all the fetal vessels enabling increased blood flow. ${ }^{5,8}$

The mean PI in the current study for FGR fetuses was $2.8 \pm 0.37$ and $2.2 \pm 0.33$ at 28-31 weeks and 28-34 weeks (Table 2). ${ }^{9-12}$ The major change in fetal hemodynamics of FGR was postulated to be the phenomenon of cerebral redistribution, which was in turn responsible for the reduced blood supply of relatively unimportant organs (liver, kidneys, limbs) and increased blood flow to the major life-saving organs of the fetus (heart, brain, adrenals). ${ }^{7}$ However, these changes were again studied in detail and the likely sequence of events were described based on the Doppler studies of various vessels. $^{7}$

All the studies point to the fact that FGR does not happen at once but usually follows certain stages and hence the struggle to establish the best, earliest, and the most reproducible predictor. ${ }^{1}$ Fetal renal artery Doppler studied by most as a predictor of FGR has shown significant association. There was a fall in blood supply to kidney with raise in the resistance in the advanced stages of FGR. However, in this study the cases of FGR were in their initial stage. Usually in initial stages of FGR or mild placental disease, there is an increased blood flow to all the organs irrespective of the body's need to compensate the hypoxia of placental dysfunction. ${ }^{13}$ This is usually not evident till a certain extent in the umbilical arteries. Hence, umbilical arteries are not stand-alone markers of FGR. ${ }^{2}$ At this stage, there will not be any recordable change in MCA PI also. Hence, at very early stages of FGR, where the abdominal circumference or estimated fetal weight has reached 10th centile without Doppler changes, renal artery Doppler can be used to differentiate it from constitutionally small fetuses to FGR, which helps in further management and prognosis. ${ }^{14}$

Analysis of oligoamnios and renal artery Doppler PI both at 28-31 weeks and 32-34 weeks showed statistically significant association. There was increased blood flow in cases with oligoamnios to compensate for the reduced liquor. This explains the decreased PI in fetuses with oligoamnios. In this study, it has also been observed that in a few fetuses with very severe oligohydramnios, there was also an increase in resistance in blood flow. ${ }^{15}$ The overall mean PI in cases of oligohydramnios has shown reduced $\mathrm{Pl}$, as cases of severe oligoamnios were very few (Table 3 ). This finding was consistent with other studies, which could not find a correlation between renal blood flow and oligohydramnios, suggesting the other mechanisms play a major role in oligohdyrmanios. ${ }^{16-20}$
Table 3: Analysis of renal artery Doppler (RAD) and umbilical artery Doppler (UAD) in fetuses with oligoamnios

\begin{tabular}{lclll}
\hline $\begin{array}{l}\text { Oligohydramnios } \\
(n=48)\end{array}$ & Mean \pm 2SD & $p$ value & $\begin{array}{l}\text { Sensitivity } \\
(\%)\end{array}$ & $\begin{array}{l}\text { Specificity } \\
(\%)\end{array}$ \\
\hline RAD 28w PI & $3.07 \pm 0.3$ & 0.0002 & 60.42 & 70.52 \\
RI & $0.91 \pm 0.20$ & 0.067 & 41.67 & 78.61 \\
RAD 34w PI & $2.3 \pm 0.29$ & 0.0047 & 68.75 & 60.69 \\
RI & $0.85 \pm 0.20$ & 0.19 & 62.5 & 57.51 \\
UAD 28w PI & $0.86 \pm 0.14$ & 0.99 & 41.67 & 72.83 \\
RI & $0.76 \pm 0.29$ & 0.0006 & 85.42 & 44.22 \\
UAD 34w PI & $0.74 \pm 0.11$ & 0.33 & 27.08 & 84.68 \\
RI & $0.68 \pm 0.27$ & 0.0003 & 83.33 & 44.22 \\
\hline
\end{tabular}

$\mathrm{RI}$, resistivity index

Table 4: Analysis of renal artery Doppler (RAD) and umbilical artery Doppler (UAD) in preterm fetuses

\begin{tabular}{lrlll}
\hline $\begin{array}{l}\text { Preterm } \\
n=48\end{array}$ & Mean \pm 2 SD & p value & $\begin{array}{l}\text { Sensitivity } \\
(\%)\end{array}$ & $\begin{array}{l}\text { Specificity } \\
(\%)\end{array}$ \\
\hline RAD 28w PI & $2.8 \pm 0.34$ & 0.0001 & 60.87 & 73.96 \\
RI & $0.97 \pm 0.18$ & 0.027 & 76.09 & 42.31 \\
RAD 34w PI & $2.2 \pm 0.28$ & 0.04 & 56.62 & 71.6 \\
RI & $0.85 \pm 0.10$ & 0.7 & 54.3 & 56.2 \\
UAD 28w PI & $0.86 \pm 0.15$ & 0.56 & 43.38 & 72.19 \\
RI & $0.62 \pm 0.11$ & 0.34 & 34.78 & 76.33 \\
UAD 34w PI & $0.76 \pm 0.1$ & 0.353 & 32.61 & 78.11 \\
RI & $0.69 \pm 0.18$ & 0.115 & 63.04 & 55.03 \\
\hline
\end{tabular}

$\mathrm{RI}$, resistivity index

In the present study, there was also a significant correlation between spontaneous preterm deliveries and renal artery Doppler indices (Table 4). There was an increase in blood flow to kidney compared to the other fetuses. In studies done on spontaneous preterm labor, infection of amniotic membranes is one of the major explanations given. ${ }^{21}$ There were also studies showing elevated inflammatory markers in cases of spontaneous preterm labor. These inflammatory markers affect kidney in a biphasic pattern, first phase with increased blood flow and the second with reduced blood flow. Another explanation is that it could also be due to dysregulated vasodilators in response to the inflammatory mediators. The same has been discussed in a similar study conducted by Humberto et al. ${ }^{21}$ However, they could not establish any relationship with renal artery Doppler and preterm birth. This event can have a genetic predisposition and other multifactorial causes may be involved warranting need for further studies.

In the present study, 62 women had high-risk factors identified such as diabetes, hypertension, previous recurrent pregnancy loss, and advanced maternal age that may have a role in affecting fetal hemodynamics. When the renal artery and umbilical artery Doppler parameters were studied individually in low-risk and high-risk groups, there was no significant change in impedance between both the groups. The risk factors in the study population were not severe enough to cause significant increase in impedance in the peripheral arterial flow and cause redistribution. Similar studies conducted on preeclampsia and its association with renal artery Doppler showed mixed results. ${ }^{22}$ The explanations given in these cases were increased thromboxane A2 and prostacyclin C 
in preeclampsia, placental disease causing similar hemodynamic changes as seen in FGR.

There was also no significant difference noted in the mode of delivery with renal artery Doppler indices. With all these antenatal associations, the perinatal outcome evaluated in terms of NICU admissions can also be predicted with renal artery Doppler indices, and PI performed better than RI.

\section{Drawbacks}

Renal volume, parenchymal thickness, and peak systolic velocities were not studied in the present study. A larger study with randomization involving both high risk and low risk would improve both the quality and quantity of data.

\section{Conclusion}

Renal artery Doppler indices were significantly more reliable in predicting adverse perinatal outcomes than umbilical artery Doppler indices. Additionally, renal artery Doppler indices were able to pick up these changes earlier, hence can be a potential early indicator, and PI being more specific and sensitive.

\section{Further Research}

It is well-known that cases of mild FGR are also associated with poor perinatal outcome and are missed more often than the cases with severe FGR especially in countries like India, where most of the obstetricians in the rural areas still use only clinical examination in assessing the fetal growth. In this study, a newer marker has been identified in significant cases with mild presentation of uteroplacental insufficiency. Hence, further research into fetal renal artery Doppler with a larger population and larger studies involving cases and controls is required.

\section{LIMITATIONS}

The sample population consisted majority of the cases with mild presentation limited by our exclusion criteria to take Doppler values twice during the study. Hence, we could not study these parameters in cases with severe uteroplacental insufficiency. Sample size of the study is limited. Larger studies are required. Additionally, patients at a higher risk for FGR in comparison with low-risk cases should be studied with more sample size as this study did not show any significant association with comorbidities.

\section{References}

1. Figueras $F, G$ ratacos E. Update on the diagnosis and classification of fetal growth restriction and proposal of a stage-based management protocol. Fetal Diagn Ther 2014;36(2):86-98. DOI: 10.1159/000357592.

2. Oros D, Figueras F, Cruz-Martinez $R$, et al. Longitudinal changes in uterine, umbilical and fetal cerebral Doppler indices in late-onset small-for-gestational age fetuses. Ultrasound Obstet Gynecol 2011;37(2):191-195. DOI: 10.1002/uog.7738.

3. Hecher K, Spernol R, Szalay S. Doppler blood flow velocity waveforms in the fetal renal artery. Arch Gynecol Obstet 1989;246(3):133-137. DOI: $10.1007 /$ BF00934074.

4. El-Aal A, Maged H, El-Sheikha KZ, et al. Fetal renal volume and renal artery Doppler in normal and intrauterine growth restricted fetuses. Egypt J Hosp Med 2018;73(3):6238-6242.
5. Arduini D, Rizzo G. Normal values of pulsatility index front fetal vessels: a cross-sectional study on 1556 healthy fetuses. J Perinat Med-Offici J Wapm 1990;18(3):165-172. DOI: 10.1515/jpme.1990.18.3.165.

6. Andriani G, Persico A, Tursini S, et al. The renal-resistive index from the last 3 months of pregnancy to 6 months old. BJU Int 2001;87(6):562564. DOI: 10.1046/j.1464-410X.2001.00085.x.

7. Ponglopisit S, Hanprasertpong T, Geater A, et al. Doppler waveform patterns and reference ranges of fetal renal artery blood flow indices in normal Thai fetuses during the second trimester. Asian Biomed 2017;9(2):217-224.

8. Vyas S, Nicolaides KH, Campbell S. Renal artery flow-velocity waveforms in normal and hypoxemic fetuses. Am J Obstet Gynecol 1989;161(1):168-172. DOI: 10.1016/0002-9378(89)90257-3.

9. Tanabe R. Doppler ultrasonographic assessment of fetal renal artery blood flow velocity waveforms in intrauterine growth retarded fetuses. Kurume Med J 1992;39(3):203-208. DOI: 10.2739/ kurumemedj.39.203.

10. Arduini D, Rizzo G. Fetal renal artery velocity waveforms and amniotic fluid volume in growth-retarded and post-term fetuses. Obstet Gynecol 1991;77(3):370-373.

11. Yoshimura $\mathrm{S}$, Masuzaki $\mathrm{H}$, Gotoh $\mathrm{H}$, et al. Fetal redistribution of blood flow and amniotic fluid volume in growth-retarded fetuses. Early Hum Dev 1997;47(3):297-304. DOI: 10.1016/S0378-3782(96)01798-7.

12. Akita A, Okada O, Saito T, et al. Evaluation of the renal artery in the fetuses with growth retardation and oligohydramnios by two dimensional Doppler ultrasonography. Nihon Sanka Fujinka Gakkai zasshi 1991;43(11):1554-1560.

13. Terstappen F, Paauw ND, Alderliesten $T$, et al. Elevated renal tissue oxygenation in premature fetal growth restricted neonates: an observational study. PLoS ONE 2018;13(9):e0204268. DOI: 10.1371/ journal.pone.0204268.

14. Benavides-Serralde A, Scheier M, Cruz-Martinez R, et al. Changes in central and peripheral circulation in intrauterine growth-restricted fetuses at different stages of umbilical artery flow deterioration: new fetal cardiac and brain parameters. Gynecol Obstet Invest 2011;71(4):274-280. DOI: 10.1159/000323548.

15. Fong K, Ryan ML, Cohen $\mathrm{H}$, et al. Doppler velocimetry of the fetal middle cerebral and renal arteries: interobserver reliability. J Ultrasound Med 1996;15(4):317-2153. DOI: 10.7863/jum.1996.15.4.317.

16. Akram W. The role of resistance index in fetal renal artery in causing oligohydramnios among overdue pregnant women. Mustansiriya Medical Journal 2018;13(1):6.

17. Sanad ZF, Gaied AM, Dawod RM, et al. Predictive value of fetal renal artery Doppler indices in idiopathic oligohydramnios and polyhydramnios. Menoufia Med J 2019;32(2):476. DOI: 10.4103/mmj. mmj_220_18.

18. Özkan MB, Özkan E, Emiroglu B, et al. Doppler study of the fetal renal artery in oligohydramnios with post-term pregnancy. J Med Ultrasound 2014;22(1):18-21. DOI: 10.1016/j.jmu.2013.10.005.

19. Figueira CO, Surita FG, Dertkigil MS, et al. Longitudinal reference intervals for Doppler velocimetric parameters of the fetal renal artery correlated with amniotic fluid index among low-risk pregnancies. Int J Gynecol Obstet 2015;131(1):45-48. DOI: 10.1016/j.ijgo.2015.05.010.

20. Budunoglu MD, Yapca OE, Yldildiz GA, et al. Fetal renal blood flow velocimetry and cerebro-placental ratio in patients with isolated oligohydramnios. J Gynecol Obstetr Human Reproduc 2019;48(7):495-499. DOI: 10.1016/j.jogoh.2019.06.002.

21. Azpurua H, Dulay AT, Buhimschi IA, et al. Fetal renal artery impedance as assessed by Doppler ultrasound in pregnancies complicated by intraamniotic inflammation and preterm birth. Am J Obstet Gynecol 2009;200(2):203-e1. DOI: 10.1016/j.ajog.2008.11.001.

22. Ma'ayeh M, Krishnan V, Gee SE, et al. Fetal renal artery impedance in pregnancies affected by preeclampsia. J Perinat Med 2020(4):(aheadof-print) 10.1515/jpm-2020-0024. 